\title{
Antiproton-Driven Fusion Propulsion System for OTV Applications
}

\author{
Terry Kammash ${ }^{1}$ and Ricky Tang ${ }^{2}$ \\ University of Michigan, Ann Arbor, MI, 48109
}

\begin{abstract}
[Abstract] It has often been argued that fusion propulsion systems are strictly suitable for deep space missions such as those to the outer planets or interstellar space due to the large specific impulses they are capable of producing. It is intriguing however to find out how suitable they may be for near Earth missions such as orbit transfers from low Earth orbits (LEO) to geosynchronous orbits (GEO). We examine these cases using an antiprotondriven fusion system consisting of a Gasdynamic Mirror (GDM) magnetic confinement chamber connected to an antiproton "trap". Taking LEO to be at an altitude of $200 \mathrm{~km}$ and GEO to be at approximately $36,000 \mathrm{~km}$, we follow Cassenti's analysis ${ }^{1}$ of Hohmann transfers to establish the required $\Delta v$ for a basic mission of transferring a vehicle from LEO to GEO and back. It is found that $\Delta v=5.5 \mathrm{~km} / \mathrm{sec}$. The vehicle in question is the DT burning fusion propulsion system noted above where plasma heating in the device is achieved by the fission fragments and annihilation products resulting from the "at rest" annihilation of antiprotons in $\mathrm{U}^{238}$ targets. It is found that such a system will produce an Isp of $2 \times 10^{5}$ seconds and a thrust of $1.2 \times 10^{6}$ Newtons for a total mass of approximately $23 \times 10^{3} \mathrm{mT}$. Assuming a continuous burn acceleration/deceleration type of trajectory, we find that the orbital transfer noted above can be undertaken in about 8 hours, and the amount of antiprotons needed is about $4 \mu \mathrm{g}$. The propulsion system will have a specific power of about 40 and a thrust to weight ratio of about $5 \times 10^{-3}$.
\end{abstract}

\section{Nomenclature}

$\begin{array}{ll}\beta & =\text { ratio of plasma pressure to vacuum magnetic field pressure } \\ L & =\text { plasma length } \\ \lambda & =\text { collision mean free path } \\ n & =\text { electron density } \\ R & =\text { plasma mirror ratio } \\ R_{0} & =\text { vacuum mirror ratio } \\ T & =\text { electron temperature } \\ \tau_{c} & =\text { plasma confinement time } \\ v_{t h} & =\text { ion thermal velocity }\end{array}$

\section{Introduction}

SEVERAL studies ${ }^{2,3}$ in recent years have shown that "at rest" annihilation of antiprotons in the Uranium isotope $\mathcal{U} \mathrm{U}^{238}$ leads to fission at nearly $100 \%$ efficiency. The resulting highly charged, fast fission fragments are highly ionizing, and can heat a suitable medium to very high temperatures. When an antiproton or a proton with multiple $\mathrm{MeV}$ kinetic energy slams into a target material it undergoes collisions with the electrons of the target and slows down by giving up energy to these particles. In the case of the proton it comes to rest in the material and forms a chemical bond with other atoms or diffuses around as atomic hydrogen. In the case of the antiproton it displaces an orbital electron around the nucleus, and begins immediately to cascade down in energy towards the ground state emitting $\mathrm{x}$-rays as it makes these transitions. Eventually it enters the nucleus and an annihilation with either a

\footnotetext{
${ }^{1}$ Professor Emeritus, Dept. of Nuclear Engineering and Radiological Sciences, 2355 Bonisteel Blvd., Ann Arbor, MI 48109, AIAA Associate Fellow.

${ }^{2}$ Graduate Student, Dept. of Aerospace Engineering, 1320 Beal Ave., Ann Arbor, MI 48109.
} 
neutron or a proton takes place. At this point the kinetic energy of the antiproton is measured in $\mathrm{eV}$, and not in $\mathrm{MeV}$, hence the label "at rest" annihilation. Nuclear fission following the annihilation at rest of antiprotons in heavy nuclei has been demonstrated in Uranium and Bismuth, and measurements have been made of the mass distribution of the fission fragments, as well as the multiplicity of the light charged particles that were emitted in the process. It was shown for example ${ }^{4}$ that in the case of uranium the average masses and kinetic energies of the fission fragments are respectively $212 \mathrm{amu}$ and $160 \mathrm{MeV}$. It was also shown that, on the average, the fission fragments left the target nearly isotropically, making it especially desirable for heating a propellant. We utilize this information to propose a propulsion device that can readily be used for orbit transfer missions.

The proposed propulsion system, illustrated in Fig. 1, consists of an antiproton "trap" attached to a gasdynamic mirror (GDM) magnetic confinement device that will confine the hydrogen $(\mathrm{H})$ plasma while being heated by the annihilation process. This heating can occur in one of two approaches: i) "at rest" annihilation of antiprotons in $\mathrm{U}^{238}$ nuclei with the resulting fission fragments depositing their energy in the hydrogen propellant or ii) the annihilation of the antiprotons ( $\bar{p}$ 's) on the protons ( $p$ 's) in the hydrogen ions with the energy of the annihilation products providing the source of heating. The first approach has the distinct advantage of the significant contribution the fission fragments can make to the thrust generated by the system resulting from their large mass. Moreover, while the fission fragments may provide the bulk of the heating, the annihilation products that are produced simultaneously will also contribute in spite of their relatively short lifetimes. In short, it appears that the first approach could be more effective not only from the propulsion standpoint, but more importantly, from the standpoint of requiring smaller amounts of antiprotons to accomplish the same objective. The underlying principle in both instances is the fact that when an antiproton annihilates on a proton (inside a nucleus or free), very energetic positively and negatively charged pions are produced in addition to charge-neutral pions which decay instantly into gamma rays. The charged pions also decay in about 72 nanoseconds into charged, energetic muons which in turn decay in $6.2 \mu$ s into electrons, positrons and a variety of neutrinos. The pions and the muons are the annihilation products that do play a role in the heating mechanisms alluded to above.

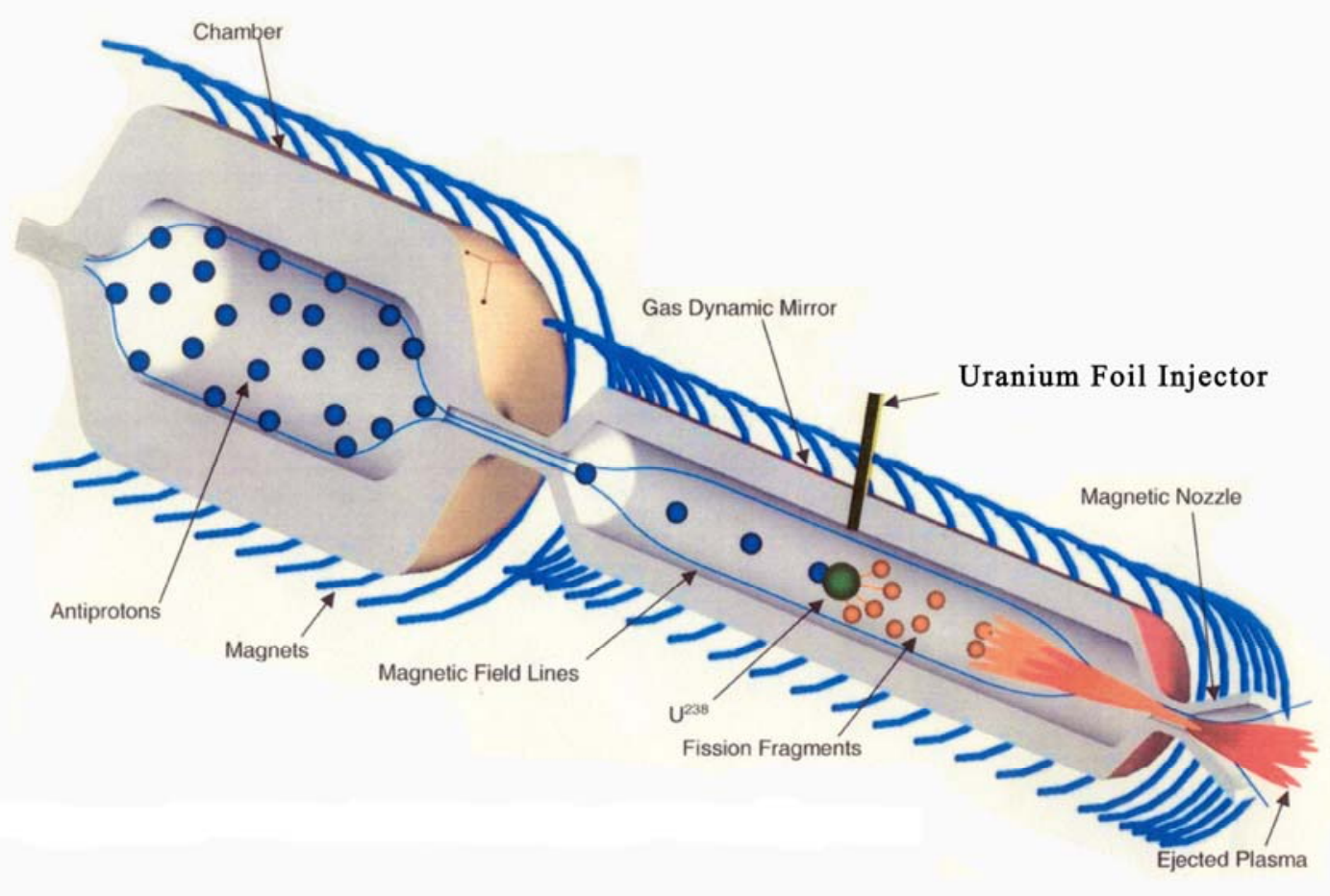

Figure 1. The proposed antiproton-driven propulsion system. 


\section{Propulsion Device}

As illustrated in Fig. 1, the proposed system consists of an antiproton housing chamber "trap" which can be made to pulse a beam of antiprotons into the magnetic bottle where it will strike a radially injected $\mathrm{U}^{238}$ target, perhaps in the form of a foil, at a pre-determined position to induce fission. The resulting highly charged, energetic fission fragments are also highly ionizing, and can heat a background of hydrogen $(\mathrm{H})$ plasma - inserted into the magnetic chamber just prior to the annihilation reaction - to a high temperature such as $1 \mathrm{keV}$. A particularly suitable magnetic bottle is an "asymmetric" gasdynamic mirror ${ }^{5}$ (GDM) where the asymmetry allows for ejection from one mirror that serves as a magnetic "nozzle" in preference to the opposite mirror through which the antiprotons are allowed to enter. Asymmetry as well as proper pulsing will allow this to happen so as to guard against any of the plasma components entering the antiproton trap. Although the fission fragments may provide the bulk of the heating, the pions with kinetic energies of $250 \mathrm{MeV}$ each, and the muons with individual energies of about 192.3 MeV also contribute significantly in spite of the short lifetimes noted earlier.

The underlying confinement principle of GDM is that the plasma be of such density and temperature as to make the ion-ion collision mean free path much shorter than its length. Under these conditions the plasma behaves much like a fluid, and its escape from the system would be analogous to the flow of a gas into vacuum from a vessel with a hole, a desirable characteristic for a thruster. In fact, the condition for plasma confinement in GDM is given by ${ }^{6}$

$$
\frac{\lambda}{R}<<L
$$

where $\lambda$ is the collision mean free path, $L$ the length of the plasma, and $R$ the plasma mirror ratio defined by

$$
R=\frac{R_{0}}{\sqrt{1-\beta}}
$$

In the above expression, $R_{0}$ denotes the vacuum mirror ratio, and $\beta$ denotes the ratio of the plasma pressure to the (vacuum) magnetic field pressure. An expression of $\lambda$ appropriate for the system under consideration can be written as

$$
\lambda(\mathrm{cm})=1.25 \times 10^{18} \frac{T^{2}(\mathrm{keV})}{n\left(\mathrm{~cm}^{-3}\right)}
$$

where a plasma density of $10^{16} \mathrm{~cm}^{-3}$ and a temperature of $1 \mathrm{keV}$ and a large plasma mirror ratio as obtained from $\beta \sim$ 0.90 will readily satisfy Eq. (1) for a device several meters in length. The large value of $\beta$ assumed here is consistent with experimental results ${ }^{7}$ that reveal an effective hot plasma confinement with $\beta \sim 1$ in a GDM device where no large scale instabilities of any sort that could lead to a rapid breakups of the plasma were detected. Moreover, an explicit expression for plasma confinement in GDM can be shown to have the form ${ }^{8}$

$$
\tau_{c}=\frac{R L}{v_{t h}}
$$

where $v_{t h}$ is the mean velocity of the ions which ultimately dictates the specific impulse produced by the system. It is interesting to note from Eq. (4) the dependence of the confinement time in GDM on the plasma mirror ratio $R$ rather than on its logarithm as in the case with the "collisionless" mirror. Also in contrast to the collisionless mirror, $\tau_{c}$ varies inversely with the square root of the ion energy rather than directly with the energy to the 3/2 power, and directly on $L$ which is totally absent in the collisionless mirror case. The dependence on length in GDM is particularly significant since it provides an important parameter that can be appropriately manipulated to ensure its suitability for propulsion application.

We envision the proposed device shown in Fig. 1 to function as a propulsion system in accordance with the following steps. A foil containing $\mathrm{U}^{238}$ is radially inserted into the GDM chamber at the exact position where an axially injected antiproton beam (of certain energy such as $20 \mathrm{keV}$ ) is calculated to effectively come to rest. Upon inducing fission in the target, the fission fragments will emerge approximately isotropically in the background of 
relatively cold (with a temperature comparable to the ionization energy) hydrogen plasma and begin to interact primarily with the electrons of this plasma giving up their energy to these particles in a predictable time period. These electrons will subsequently transfer this energy through thermalization with the ion component, and the time characterizing this transfer will dictate the minimum confinement time demanded of the GDM machine as noted in Eq. (4). With proper choice of the number of incident antiprotons and uranium target density and dimensions, sufficient energy from the fission fragments as well as the annihilation products can indeed be deposited to heat the plasma to the desired temperature in the prescribed confinement time.

Preliminary calculations reveal that such a GDM propulsion device with an aspect ratio (length/diameter) that ensures stability against curvature-driven MHD modes (while simultaneously satisfying the condition prescribed by Eq. (1) can produce specific impulses of $10^{4}-10^{5}$ seconds at thrusts of tens of kilo-Newtons. It should also be noted that the presence of the mirror ratio $R$ in Eq. (1) guards against "loss cone" instabilities especially at large values since that represents effective closing of this domain in velocity space. Finally, the high plasma "collisionality" characterizing GDM confinement represents another stabilizing factor for "micro-instabilities" driven by temperature anisotropy which if allowed to exist could lead to turbulence and enhanced plasma diffusion across magnetic field lines.

\section{Sample Orbit Transfer Mission (OTM)}

As a measure of the propulsive capability of the proposed system, an analysis has been carried out of an orbit transfer mission for a vehicle from LEO to GEO and back to LEO to establish the relevant parameters that define a system that can be utilized for orbit transfer. The results are shown in Table 1.

Table 1. System characteristics for a DT fusion system.

\begin{tabular}{|ll||ll|}
\hline Plasma Density & $5 \times 10^{17} \mathrm{~cm}^{-3}$ & Specific Impulse & $2.0 \times 10^{5} \mathrm{sec}$ \\
Ion Temperature & $10 \mathrm{keV}$ & Thrust & $1.2 \times 10^{6} \mathrm{~N}$ \\
Plasma Radius & $4.5 \mathrm{~cm}$ & System Mass & $23 \times 10^{3} \mathrm{Mg}$ \\
Plasma Length & $20 \mathrm{~m}$ & Amount of Antiprotons & $4 \mu \mathrm{g}$ \\
\hline
\end{tabular}

\section{Conclusion}

The various steps involved in the operation of the propulsion device, described above, dictate the major technical issues to be addressed. Once addressed, they will provide a comprehensive analytic understanding on which to base a meaningful design of a future GDM propulsion system driven by antiprotons.

Antiprotons are produced by smashing protons into a metallic target. There are only a few government laboratories around the world where antiprotons can be produced in any meaningful quantities. By far the most intense source of antiprotons is the Fermi National Accelerator Laboratory (FNAL or Fermilab) in Batavia, Illinois.

Currently, Fermilab produces $5 \times 10^{14}$ antiprotons per year. The Fermilab complex consists of a sequence of accelerator stages, initially linacs merging into synchrotrons, that produce the antiprotons and then accelerate them to very relativistic velocities. One of the stages, the Main Injector, contains the antiproton beam at a momentum of $9 \mathrm{GeV} / \mathrm{c}$. It has been noted that the components of the Main Injector can act as a decelerator of the antiprotons. By reducing the momentum to below $2 \mathrm{GeV} / \mathrm{c}$, it will be possible to utilize standard degrading technology or another stage of deceleration to reduce the energy of the particles to as low as $20 \mathrm{keV}$. At $20 \mathrm{keV}$, the particles can be injected and trapped in a Penning Trap. The operational cost of producing antiprotons has recently been estimated by Fermilab to be $\$ 27.4 \mathrm{M}$ per year. Thus, the cost per $10^{10}$ is about $\$ 550$.

Storage of antiprotons has been demonstrated repeatedly. Penning Traps have been used for decades to hold clouds of charged particles. Typical applications have centered around holding ions or atoms in a vacuum for sensitive analysis. Recently, Penning Traps have been used to cool clouds of atoms down to ultracold temperatures to create "superatoms" that can be studied via laser interactions. Within the past two decades, however, Penning Traps have been built to hold antimatter. ${ }^{9,10}$

In essence, a Penning Trap is a hollow pipe that contains a very hard vacuum. The pipe is centered in a uniform magnetic field so that the field runs parallel to the pipe axis. Electrode rings inside the pipe create an electric field "basket" in which charged particles will accumulate and stay. The electric field creates a region in the center of the trap that holds the charged particles. Any sideways motion by the particles causes them to spiral around the 
magnetic field lines. Thus, they are prevented from hitting the walls of the pipe. For trapping antiprotons, the vacuum that can be obtained in the trap will determine the lifetime of the storage.

\section{References}

${ }^{1}$ Cassenti, B. N., Journal of Propulsion and Power, Vol. 1, No. 2, 1985, pp. 143.

${ }^{2}$ Hoffman, P., et al., Physical Review C, Vol. 49, 1994, pp. 2555.

${ }^{3}$ Kim, Y. S., et al., Physical Review C, Vol. 54, 1996, pp. 2469.

${ }^{4}$ Bocquet, J. P., et al., Z. Phys. A, Hadrons and Nuclei, Vol. 342, 1992, pp. 183.

${ }^{5}$ Kammash, T., and Lee, M. J., Journal of Propulsion and Power, Vol. 11, 1995, pp. 544.

${ }^{6}$ Nagornyv, V. P., et al., Nuclear Fusion, Vol. 24, 1984, pp. 1421.

${ }^{7}$ Zhitlukhin, A. M., et al., JETP Letters, Vol. 39, 1984, pp. 293. (AIP English version)

${ }^{8}$ Mirnov, V. V. and Ryutov, D. D., Soviet Tech. Phys. Letters, Vol. 5, 1979, pp. 279.

${ }^{9}$ Hynes, M. V., Picklesimer, A., and Robertson, W. G., "Vacuum Requirements for Antiproton, Proton, and $\mathrm{H}^{-}$Ion Storage in Penning Traps,” Los Alamos National Laboratory document LA-UR-87-3054.

${ }^{10}$ Howe, S. D., Hynes, M. V., and Picklesimer, A., "Portable Pbars: Traps That Travel," Proceedings of the $2^{\text {nd }}$ Workshop on Antiproton Technology, Santa Monica, CA, October 1987. 